\title{
The first nontrivial curve in the fučlk spectrum of the dirichlet laplacian on the ball consists of nonradial eigenvalues
}

\author{
Jiřl Benedikt ${ }^{*}$, Pavel Drábek ${ }^{2}$ and Petr Girg ${ }^{1}$
}

\author{
* Correspondence: benedikt@kma. \\ zcu.cz \\ ${ }^{1}$ Department of Mathematics, \\ Faculty of Applied Sciences, \\ University of West Bohemia, \\ Univerzitnĺ 22, 30614 Plzeň, Czech \\ Republic \\ Full list of author information is \\ available at the end of the article
}

\begin{abstract}
It is well-known that the second eigenvalue $\lambda_{2}$ of the Dirichlet Laplacian on the ball is not radial. Recently, Bartsch, Weth and Willem proved that the same conclusion holds true for the so-called nontrivial (sign changing) Fučík eigenvalues on the first curve of the Fučík spectrum which are close to the point $\left(\lambda_{2}, \lambda_{2}\right)$. We show that the same conclusion is true in dimensions 2 and 3 without the last restriction.
\end{abstract}

Keywords: Fučík spectrum, The first curve of the Fučík spectrum, Radial and nonradial eigenfunctions

\section{Introduction}

Let $\Omega \subset \mathbb{R}^{N}$ be a bounded domain, $N \geq 2$. The Fučík spectrum of $-\Delta$ on $W_{0}^{1,2}(\Omega)$ is defined as a set $\Sigma$ of those $\left(\lambda_{+}, \lambda_{-}\right) \in \mathbb{R}^{2}$ such that the Dirichlet problem

$$
\begin{cases}-\Delta u=\lambda_{+} u^{+}-\lambda_{-} u^{-} & \text {in } \Omega, \\ u=0 & \text { on } \partial \Omega\end{cases}
$$

has a nontrivial solution $u \in W_{0}^{1,2}(\Omega)$. In particular, if $\lambda_{1}<\lambda_{2}<\ldots$ are the eigenvalues of the Dirichlet Laplacian on $\Omega$ (counted with multiplicity), then clearly $\Sigma$ contains each pair $\left(\lambda_{k}, \lambda_{k}\right), k \in \mathbb{N}$, and the two lines $\left\{\lambda_{1}\right\} \times \mathbb{R}$ and $\mathbb{R} \times\left\{\lambda_{1}\right\}$. Following [1, p. 15], we call the elements of $\Sigma \backslash\left(\left\{\lambda_{1}\right\} \times \mathbb{R} \cup \mathbb{R} \times\left\{\lambda_{1}\right\}\right)$ nontrivial Fuč $i k$ eigenvalues. It was proved in [2] that there exists a first curve $\mathcal{C}$ of nontrivial Fučík eigenvalues in the sense that, defining $\eta:\left(\lambda_{1}, \infty\right) \rightarrow \mathbb{R}$ by

$$
\eta(\lambda) \stackrel{\text { def }}{=} \inf \left\{\mu>\lambda_{1}:(\lambda, \mu) \text { is a nontrivial Fučík eigenvalue }\right\},
$$

we have that $\lambda_{1}<\eta(\lambda)<\infty$ for every $\lambda\left(>\lambda_{1}\right)$, and the curve

$$
\mathcal{C} \stackrel{\text { def }}{=}\left\{(\lambda, \eta(\lambda)): \lambda \in\left(\lambda_{1}, \infty\right)\right\}
$$

consists of nontrivial Fučík eigenvalues. Moreover, it was proved in [2] that $\mathcal{C}$ is a continuous and strictly decreasing curve which contains the point $\left(\lambda_{2}, \lambda_{2}\right)$ and which is symmetric with respect to the diagonal.

It was conjectured in [1, p. 16], that if $\Omega$ is a radially symmetric bounded domain, then every eigenfunction $u$ of $(1)$ corresponding to some $\left(\lambda_{+}, \lambda_{-}\right) \in \mathcal{C}$ is not radial. The

(c) 2011 Benedikt et al; licensee Springer. This is an Open Access article distributed under the terms of the Creative Commons Attribution License (http://creativecommons.org/licenses/by/2.0), which permits unrestricted use, distribution, and reproduction in any medium, provided the original work is properly cited. 
authors of $\left[1\right.$, p. 16] actually proved that the conjecture is true if $\left(\lambda_{+}, \lambda_{-}\right) \in$ Cbut sufficiently close to the diagonal.

The original purpose of this paper was to prove that the above conjecture holds true for all $\left(\lambda_{+}, \lambda_{-}\right) \in \mathcal{C}$ provided $\Omega$ is a ball in $\mathbb{R}^{N}$ with $N=2$ and $N=3$. Without loss of generality, we prove it for the unit ball $B$ centred at the origin. Cf. Theorem 6 below.

During the review of this paper, one of the reviewers drew the authors' attention to the paper [3], where the same result is proved for general $N \geq 2$ (see [3, Theorem 3.2]). The proof in [3] uses the Morse index theory and covers also problems with weights on more general domains than balls. On the other hand, our proof is more elementary and geometrically instructive. From this point of view, our result represents a constructive alternative to the rather abstract approach presented in [3]. This is the main authors' contribution.

\section{Variational characterization of $\mathcal{C}$}

Let us fix $s \in \mathbb{R}$ and let us draw in the $\left(\lambda_{+}, \lambda_{-}\right)$plane a line parallel to the diagonal and passing through the point $(s, 0)$, see Figure 1 .

We show that the point of intersection of this line and $\mathcal{C}$ corresponds to the critical value of some constrained functional (cf. [4, p. 214]). To this end we define the functional

$$
\mathcal{J}_{s}(u) \underline{\underline{\text { def }}} \int_{\Omega}|\nabla u|^{2}-s \int_{\Omega}\left(u^{+}\right)^{2} .
$$

Then $\mathcal{J}_{s}(u)$ is a $C^{1}$-functional on $W_{0}^{1,2}(\Omega)$ and we look for the critical points of the restriction $\tilde{\mathcal{J}}_{s}$ of $\mathcal{J}_{s}$ to

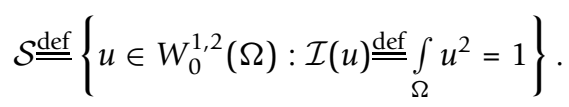

By the Lagrange multipliers rule, $u \in \mathcal{S}$ is a critical point of $\tilde{\mathcal{J}}_{s}$ if and only if there exists $t \in \mathbb{R}$ such that

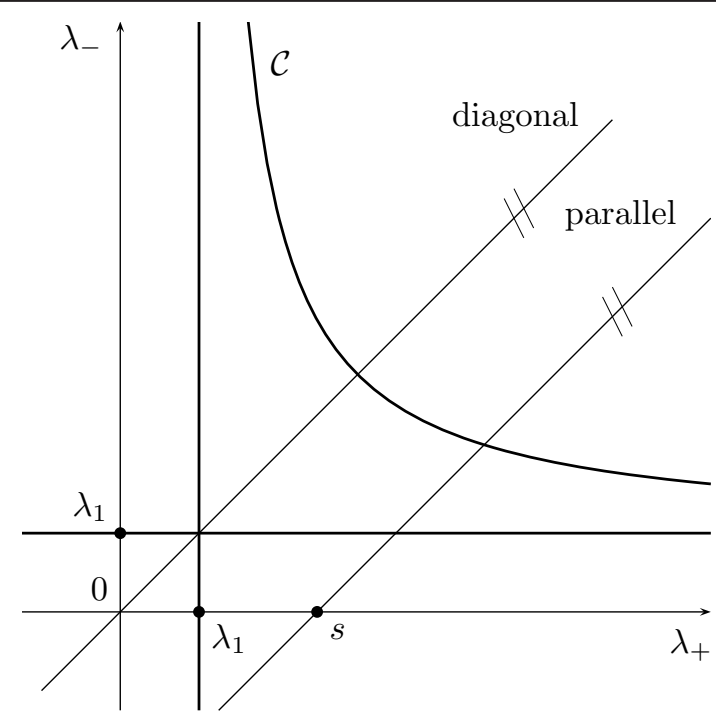

Figure 1 The first two Fučík curves. 


$$
\begin{aligned}
& \mathcal{J}_{s}^{\prime}(u)=t \mathcal{I}^{\prime}(u), \text { i.e., } \\
& \int_{\Omega} \nabla u \nabla v-s \int_{\Omega} u^{+} v=t \int_{\Omega} u v
\end{aligned}
$$

for all $v \in W_{0}^{1,2}(\Omega)$. This means that

$$
\begin{cases}-\Delta u=(s+t) u^{+}-t u^{-} & \text {in } \Omega, \\ u=0 & \text { on } \partial \Omega\end{cases}
$$

holds in the weak sense. In particular, $\left(\lambda_{+}, \lambda_{-}\right)=(s+t, t) \in \Sigma$. Taking $v=u$ in (2), one can see that the Lagrange multiplier $t$ is equal to the corresponding critical value of $\tilde{\mathcal{J}}_{s}$.

From now on we assume $s \geq 0$, which is no restriction since $\Sigma$ is clearly symmetric with respect to the diagonal. The first eigenvalue $\lambda_{1}$ of $-\Delta$ on $W_{0}^{1,2}(\Omega)$ is defined as

$$
\lambda_{1}=\lambda_{1}(\Omega) \stackrel{\underline{\text { def }}}{\min }\left\{\int_{\Omega}|\nabla u|^{2}: u \in W_{0}^{1,2}(\Omega) \text { and } \int_{\Omega}|u|^{2}=1\right\} .
$$

It is well known that $\lambda_{1}>0$, simple and admits an eigenfunction $\varphi_{1} \in W_{0}^{1,2}(\Omega) \cap C^{1}(\Omega)$ with $\phi_{1}$ satisfying $\phi_{1}(x)>0$ for $x \in \Omega$. Let

$$
\Gamma \stackrel{\text { def }}{=}\left\{\gamma \in C([-1,1], \mathcal{S}): \gamma(-1)=-\varphi_{1} \text { and } \gamma(1)=\varphi_{1}\right\}
$$

and

$$
c(s) \stackrel{\text { def }}{=} \inf _{\gamma \in \Gamma} \max _{u \in \gamma} \tilde{\mathcal{J}}_{s}(u) .
$$

We keep the same notation $\gamma$ for the image of a function $\gamma=\gamma(t)$. It follows from [4, Props. 2.2, 2.3 and Thms. 2.10, 3.1] that the first three critical levels of $\tilde{\mathcal{J}}_{s}$ are classified as follows.

(i) $\phi_{1}$ is a strict global minimum of $\tilde{\mathcal{J}}_{s}$ with $\tilde{\mathcal{J}}_{s}\left(\varphi_{1}\right)=\lambda_{1}-s$. The corresponding point in $\Sigma$ is $\left(\lambda_{1}, \lambda_{1}-s\right)$, which lies on the vertical line through $\left(\lambda_{1}, \lambda_{1}\right)$.

(ii) $-\phi_{1}$ is a strict local minimum of $\tilde{\mathcal{J}}_{s}$, and $\tilde{\mathcal{J}}_{s}\left(-\varphi_{1}\right)=\lambda_{1}$. The corresponding point in $\Sigma$ is $\left(\lambda_{1}+s, \lambda_{1}\right)$, which lies on the horizontal line through $\left(\lambda_{1}, \lambda_{1}\right)$.

(iii) For each $s \geq 0$, the point $(s+c(s), c(s))$, where $c(s)>\lambda_{1}$ is defined by the minimax formula (4), belongs to $\Sigma$. Moreover, the point $(s+c(s), c(s))$ is the first nontrivial point of $\Sigma$ on the parallel to the diagonal through $(s, 0)$.

Next we summarize some properties of the dependence of the (principal) first eigenvalue $\lambda_{1}(\Omega)$ on the domain $\Omega$. The following proposition follows immediately from the variational characterization of $\lambda_{1}$ given by (3) and the properties of the corresponding eigenfunction $\phi_{1}$.

Proposition 1. $\lambda_{1}\left(\Omega_{2}\right)<\lambda_{1}\left(\Omega_{1}\right)$ whenever $\Omega_{\dot{v}} i=1,2$, are bounded domains satisfying $\Omega_{1} \subseteq \Omega_{2}$ and meas $\left(\Omega_{1}\right)<\operatorname{meas}\left(\Omega_{2}\right)$.

Let us denote by $V_{d}, d \in(0,1)$, the ball canopy of the height $2 d$ and by $B_{d}$ the maximal inscribed ball in $V_{d}$ (see Figure 2). It follows from Proposition 1 that for $d \in(0,1)$, we have 


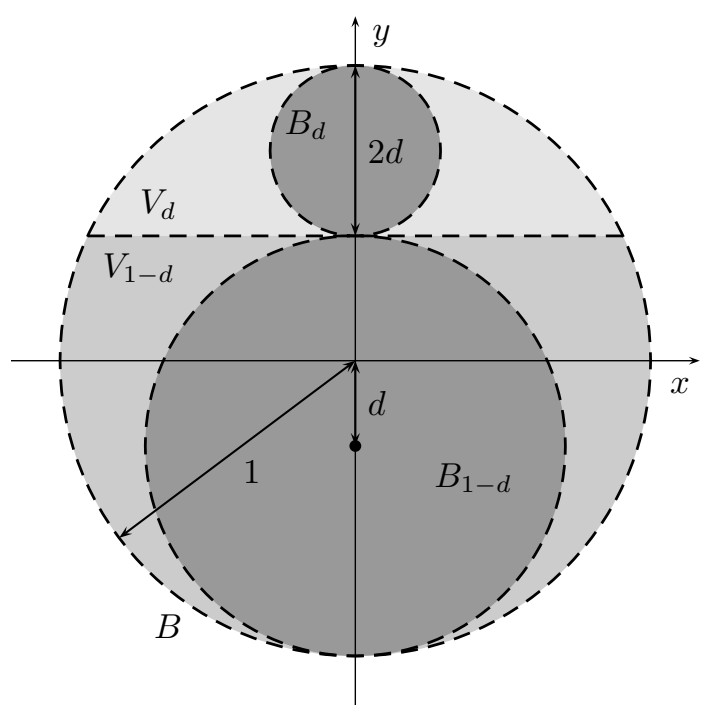

Figure 2 The ball decomposition

$$
\lambda_{1}\left(V_{d}\right)<\lambda_{1}\left(B_{d}\right), \quad \lambda_{1}\left(V_{1-d}\right)<\lambda_{1}\left(B_{1-d}\right) .
$$

Moreover, from the variational characterization (3), the following properties of the function

$$
d \mapsto \lambda_{1}\left(V_{d}\right)
$$

follow immediately.

Proposition 2. The function (6) is continuous and strictly decreasing on $(0,1)$, it maps $(0,1)$ onto $\left(\lambda_{1}(B), \infty\right)$ and $\lim _{d \rightarrow 0+} \lambda_{1}\left(V_{d}\right)=\infty, \lim _{d \rightarrow 1-} \lambda_{1}\left(V_{d}\right)=\lambda_{1}(B)$.

In particular, it follows from Proposition 2 that, given $s \geq 0$, there exists a unique $d_{s} \in\left(0, \frac{1}{2}\right]$ such that

$$
\lambda_{1}\left(V_{d_{s}}\right)=s+\lambda_{1}\left(V_{1-d_{s}}\right)
$$

Let $u_{d_{s}}$ and $u_{1-d_{s}}$ be positive principle eigenvalues associated with $\lambda_{1}\left(V_{d_{s}}\right)$ and $\lambda_{1}\left(V_{1-d_{s}}\right)$, respectively. We extend both functions on the entire $B$ by setting $u_{d_{s}} \equiv 0$ on $u_{1-d_{s}} \equiv 0, u_{1-d_{s}} \equiv 0$ on $V_{d_{s}}$ and then normalize them by $u_{d_{s}}, u_{1-d_{s}} \in \mathcal{S}$. Our aim is to construct a special curve $\gamma \in \Gamma$ on which the values of $\tilde{\mathcal{J}}_{s}$ stay below $\lambda_{1}\left(V_{d_{s}}\right)$. Actually, the curve $\gamma$ connects $\phi_{1}$ with $\left(-\phi_{1}\right)$ and passes through $u_{d_{s}}$ and $\left(-u_{1-d_{s}}\right)$. For this purpose we set $\gamma=\gamma_{1} \cup \gamma_{2} \cup \gamma_{3}$, where

$$
\begin{aligned}
& \gamma_{1} \stackrel{\text { def }}{=}\left\{u=\left(\tau \varphi_{1}^{2}+(1-\tau) u_{d_{s}}^{2}\right)^{\frac{1}{2}}: \tau \in[0,1]\right\}, \\
& \gamma_{2} \stackrel{\text { def }}{=}\left\{u=\alpha u_{d_{s}}-\beta u_{1-d_{s}}: \alpha \geq 0, \beta \geq 0, \alpha^{2}+\beta^{2}=1\right\}, \\
& \gamma_{3} \stackrel{\text { def }}{=}\left\{u=-\left(\tau \varphi_{1}^{2}+(1-\tau) u_{1-d_{s}}^{2}\right)^{\frac{1}{2}}: \tau \in[0,1]\right\} .
\end{aligned}
$$

Changing suitably the parametrization of $\gamma_{i}, i=1,2,3$ (we skip the details for the brevity), $\gamma$ can be viewed as a graph of a continuous function, mapping $[-1,1]$ into $\mathcal{S}$. We prove

Proposition 3. $\tilde{\mathcal{J}}_{s}(u) \leq \lambda_{1}\left(V_{1-d_{s}}\right)$ for all $u \in \gamma$. 
For the proof we need so-called ray-strict convexity of the functional

$$
\mathcal{J}(v) \stackrel{\text { def }}{=} \int_{\Omega}\left|\nabla v^{\frac{1}{2}}\right|^{2}
$$

defined on

$$
V_{+} \stackrel{\text { def }}{=}\left\{v: \Omega \rightarrow(0, \infty): v^{\frac{1}{2}} \in W_{0}^{1,2}(\Omega) \cap C(\bar{\Omega})\right\}
$$

We say that $\mathcal{J}: V_{+} \rightarrow \mathbb{R}$ is ray-strictly convex if for all $\tau \in(0,1)$ and $v_{1}, v_{2} \in V_{+}$we have

$$
\mathcal{J}\left((1-\tau) v_{1}+\tau v_{2}\right) \leq(1-\tau) \mathcal{J}\left(v_{1}\right)+\tau \mathcal{J}\left(v_{2}\right)
$$

where the equality holds if and only if $v_{1}$ and $v_{2}$ are colinear.

Lemma 4 (see [5, p. 132]). The functional $\mathcal{J}$ defined by (8) is ray-strictly convex.

\section{Proof of Proposition 3.}

1. The values on $\gamma_{1}$. For $u \in \gamma_{1}$ we have

$$
\begin{aligned}
\tilde{\mathcal{J}}_{s}(u) & =\mathcal{J}\left(u^{2}\right)-s \int_{B} u^{2}=\int_{B}\left|\nabla\left(\tau \varphi_{1}^{2}+(1-\tau) u_{d_{s}}^{2}\right)^{\frac{1}{2}}\right|^{2}-s \int_{B}\left(\tau \varphi_{1}^{2}+(1-\tau) u_{d_{s}}^{2}\right) \\
& \leq \tau \int_{B}\left|\nabla \varphi_{1}\right|^{2}+(1-\tau) \int_{B}\left|\nabla u_{d_{s}}\right|^{2}-s\left(\tau \int_{B} \varphi_{1}^{2}+(1-\tau) \int_{B} u_{d_{s}}^{2}\right) \\
& \leq \tau \int_{B}\left|\nabla u_{d_{s}}\right|^{2}+(1-\tau) \int_{B}\left|\nabla u_{d_{s}}\right|^{2}-s \\
& \leq \int_{V_{d_{s}}}\left|\nabla u_{d_{s}}\right|^{2}-s=\lambda_{1}\left(V_{d_{s}}\right)-s=s+\lambda_{1}\left(V_{1-d_{s}}\right)-s=\lambda_{1}\left(V_{1-d_{s}}\right)
\end{aligned}
$$

by Lemma 4 (with $\Omega:=B$ ), (3) and (7).

2. The values on $\gamma_{2}$. Let $u \in \gamma_{2}$, then there exist $\alpha \geq 0, \beta \geq 0, \alpha^{2}+\beta^{2}=1$ and such that $u=\alpha u_{d_{s}}-\beta u_{1-d_{s}}$. Since the supports of $u_{d_{s}}$ and $u_{1-d_{s}}$ are mutually disjoint, we have

$$
\begin{aligned}
\tilde{\mathcal{J}}_{s}(u) & =\alpha^{2} \int_{V_{d_{s}}}\left|\nabla u_{d_{s}}\right|^{2}+\beta^{2} \int_{V_{1-d_{s}}}\left|\nabla u_{1-d_{s}}\right|^{2}-\alpha^{2} s \int_{V_{d_{s}}} u_{d_{s}}^{2} \\
& =\alpha^{2} \lambda_{1}\left(V_{d_{s}}\right)+\beta^{2} \lambda_{1}\left(V_{1-d_{s}}\right)-\alpha^{2} s \\
& =\alpha^{2} s+\left(\alpha^{2}+\beta^{2}\right) \lambda_{1}\left(V_{1-d_{s}}\right)-\alpha^{2} s=\lambda_{1}\left(V_{1-d_{s}}\right)
\end{aligned}
$$

by (7).

3. The values on $\gamma_{3}$. For $u \in \gamma_{3}$ we have (similarly as in the first case)

$$
\tilde{\mathcal{J}}_{s}(u)=\int_{B}\left|\nabla\left(\tau \varphi_{1}^{2}+(1-\tau) u_{1-d_{s}}^{2}\right)^{\frac{1}{2}}\right|^{2} \leq \int_{V_{1-d_{s}}}\left|\nabla u_{1-d_{s}}\right|^{2}=\lambda_{1}\left(V_{1-d_{s}}\right) .
$$

From Proposition 3, (4) and (5) we immediately get

Proposition 5. Given $s \geq 0$, we have

$$
c(s) \leq \lambda_{1}\left(V_{1-d_{s}}\right)<\lambda_{1}\left(B_{1-d_{s}}\right) .
$$




\section{Radial eigenfunctions}

Radial Fučík spectrum has been studied in [6]. Let $|x|$ be the Euclidean norm of $x \in$ $\mathbb{R}^{N}$ and $u=u(|x|)$ be a radial solution of the problem

$$
\begin{cases}-\Delta u=\lambda_{+} u^{+}-\lambda_{-} u^{-} & \text {in } B \\ u=0 & \text { on } \partial B\end{cases}
$$

Set $r=|x|$ and write $v(r)=u(|x|)$. It follows from the regularity theory that (10) is equivalent to the singular problem

$$
\left\{\begin{array}{l}
v^{\prime \prime}+\frac{N-1}{r} v^{\prime}+\lambda_{+} v^{+}-\lambda_{-} v^{-}=0 \quad \text { in }(0,1), \\
v^{\prime}(0)=0, \quad v(1)=0 .
\end{array}\right.
$$

The authors of [6] provide a detailed characterization of the Fučík spectrum of (11) by means of the analysis of the linear equation associated to (11):

$$
v^{\prime \prime}+\frac{N-1}{r} v^{\prime}+\lambda v=0 \quad \text { in }(0, \infty)
$$

The function $v$ is a solution of (12) if and only if $\hat{v}(r)=r^{\frac{1}{2}(N-1)} v(r)$ is a solution of

$$
\hat{v}^{\prime \prime}+\left(\lambda+\frac{(N-1)(3-N)}{4 r^{2}}\right) \hat{v}=0 \quad \text { in }(0, \infty) .
$$

Note that the functions $v$ and $\hat{v}$ have the same zeros.

Let us investigate the radial Fučík eigenvalues which lie on the line parallel to the diagonal and which passes through the point $(s, 0)$ in the $\left(\lambda_{+}, \lambda_{-}\right)$-plane. The first two intersections coincide with the points $\left(\lambda_{1}, \lambda_{1}-s\right)$ and $\left(\lambda_{1}+s, \lambda_{1}\right)$. This fact follows from the radial symmetry of the principal eigenfunction of the Dirichlet Laplacian on the ball. A normalized radial eigenfunction associated with the next intersection has exactly two nodal domains and it is either positive or else negative at the origin. Let us denote the former eigenfunction by $u^{1}$ and the latter one by $u^{2}$, respectively. Let $\left(\lambda^{1}+\right.$ $\left.s, \lambda^{1}\right)$ and $\left(\lambda^{2}+s, \lambda^{2}\right)$ be Fučík eigenvalues associated with $u^{1}$ and $u^{2}$, respectively. The property (iii) on page 5 implies that $c(s) \leq \lambda^{i}, i=1,2$.

The main result of this paper states that the above inequalities are strict and it is formulated as follows.

Theorem 6. Let $N=2$ or $N=3$ and $s \in \mathbb{R}$ be arbitrary. Then

$$
c(s)<\lambda^{i}, \quad i=1,2 .
$$

In particular, nontrivial Fučík eigenvalues on the first curve of the Fučík spectrum are not radial.

Proof. Let $u^{i}(x)=v^{i}(r), i=1,2, r=|x|$. Then there exists $d^{1} \in(0,1)$ such that $v^{1}(r)$ is a solution of

$$
\left\{\begin{array}{l}
v^{\prime \prime}+\frac{N-1}{r} v^{\prime}+\left(s+\lambda^{1}\right) v=0 \quad \text { and } \quad v>0 \quad \text { in }\left(0, d^{1}\right) \\
v^{\prime}(0)=v\left(d^{1}\right)=0
\end{array}\right.
$$


and

$$
\left\{\begin{array}{l}
v^{\prime \prime}+\frac{N-1}{r} v^{\prime}+\lambda^{1} v=0 \quad \text { and } \quad v<0 \quad \text { in }\left(d^{1}, 1\right) \\
v\left(d^{1}\right)=v(1)=0
\end{array}\right.
$$

After the substitution $\hat{v}^{1}(r)=r^{\frac{1}{2}(N-1)} v^{1}(r), \hat{v}^{1}$ is a solution of

$$
\left\{\begin{array}{l}
\hat{v}^{\prime \prime}+\left(s+\lambda^{1}+\frac{(N-1)(3-N)}{4 r^{2}}\right) \hat{v}=0 \quad \text { and } \quad \hat{v}>0 \quad \text { in }\left(0, d^{1}\right) \\
\hat{v}(0)=\hat{v}\left(d^{1}\right)=0
\end{array}\right.
$$

and

$$
\left\{\begin{array}{l}
\hat{v}^{\prime \prime}+\left(\lambda^{1}+\frac{(N-1)(3-N)}{4 r^{2}}\right) \hat{v}=0 \quad \text { and } \quad \hat{v}<0 \quad \text { in }\left(d^{1}, 1\right) \\
\hat{v}\left(d^{1}\right)=\hat{v}(1)=0
\end{array}\right.
$$

Let $u_{1}=u_{1}(x)$ and $u_{2}=u_{2}(x)$ be the principal positive eigenfunctions associated with $\lambda_{1}\left(B_{d_{s}}\right)$ and $\lambda_{1}\left(B_{1-d_{s}}\right)$, respectively. Both $u_{i}, i=1,2$, are radially symmetric with respect to the centre of the corresponding ball. Due to the invariance of the Laplace operator with respect to translations we may assume that both $B_{d_{s}}$ and $B_{1-d_{s}}$ are centred at the origin. We then set $u_{i}(x)=w_{i}(r), i=1,2, r=|x|$. The functions $w_{i}, i=1,2$, solve

$$
\left\{\begin{array}{l}
w_{1}^{\prime \prime}{ }_{1}+\frac{N-1}{r} w_{1}^{\prime}+\lambda_{1}\left(B_{d_{s}}\right) w_{1}=0 \quad \text { and } \quad w_{1}>0 \quad \text { in }\left(0, d_{s}\right) \\
w_{1}^{\prime}(0)=w_{1}\left(d_{s}\right)=0
\end{array}\right.
$$

and

$$
\left\{\begin{array}{l}
w_{2}^{\prime \prime}{ }_{2}+\frac{N-1}{r} w_{2}^{\prime}+\lambda_{1}\left(B_{1-d_{s}}\right) w_{2}=0 \quad \text { and } \quad w_{2}>0 \quad \text { in }\left(0,1-d_{s}\right) \\
w_{2}^{\prime}{ }_{2}(0)=w_{2}\left(1-d_{s}\right)=0
\end{array}\right.
$$

After the substitution $\hat{w}_{i}(r)=r^{\frac{1}{2}(N-1)} w_{i}(r), i=1,2$, we have

$$
\left\{\begin{array}{l}
\hat{w}_{1}^{\prime \prime}+\left(\lambda_{1}\left(B_{d_{s}}\right)+\frac{(N-1)(3-N)}{4 r^{2}}\right) \hat{w}_{1}=0 \quad \text { and } \quad \hat{w}_{1}>0 \quad \text { in }\left(0, d_{s}\right) \\
\hat{w}_{1}(0)=\hat{w}_{1}\left(d_{s}\right)=0
\end{array}\right.
$$

and

$$
\left\{\begin{array}{l}
\hat{w}_{2}^{\prime \prime}+\left(\lambda_{1}\left(B_{1-d_{s}}\right)+\frac{(N-1)(3-N)}{4 r^{2}}\right) \hat{w}_{2}=0 \quad \text { and } \quad \hat{w}_{2}>0 \quad \text { in }\left(0,1-d_{s}\right) \\
\hat{w}_{2}(0)=\hat{w}_{2}\left(1-d_{s}\right)=0
\end{array}\right.
$$

The substitution $\tilde{v}(r)=-\hat{v}\left(r+d^{1}\right)$ transforms (15) to

$$
\left\{\begin{array}{l}
\tilde{v}^{\prime \prime}+\left(\lambda^{1}+\frac{(N-1)(3-N)}{4\left(r+d^{1}\right)^{2}}\right) \tilde{v}=0 \quad \text { and } \quad \tilde{v}>0 \quad \text { in }\left(0,1-d^{1}\right) \\
\tilde{v}(0)=\tilde{v}\left(1-d^{1}\right)=0
\end{array}\right.
$$

Let us assume that $\lambda^{1} \leq \lambda_{1}\left(V_{1-d_{s}}\right)\left(<\lambda_{1}\left(B_{1-d_{s}}\right)\right)$ and that $d^{1}>d_{s}$. Choose $\delta=\frac{d^{1}-d_{s}}{2}$ and set $\tilde{w}_{2}(r)=\hat{w}_{2}(r+\delta)$. Then $\tilde{w}_{2}$ solves 


$$
\left\{\begin{array}{l}
\tilde{w}_{2}^{\prime \prime}+\left(\lambda_{1}\left(B_{1-d_{s}}\right)+\frac{(N-1)(3-N)}{4(r+\delta)^{2}}\right) \tilde{w}_{2}=0 \quad \text { and } \quad \tilde{w}_{2}>0 \quad \text { in }\left(-\delta, 1-d_{s}-\delta\right) \\
\tilde{w}_{2}(-\delta)=\tilde{w}_{2}\left(1-d_{s}-\delta\right)=0 .
\end{array}\right.
$$

It follows that (18) is a Sturm majorant for (17) on the interval $\mathcal{I}=\left[-\frac{\delta}{2}, 1-d_{s}-\frac{\delta}{2}\right]$ and $\tilde{w}_{2}>0$ on $\mathcal{J}$. Since $\tilde{v}(0)=\tilde{v}\left(1-d^{1}\right)=0$ and $0 \in \mathcal{I}, 1-d^{1} \in \mathcal{I}$, we have a contradiction with the Sturm Separation Theorem (see [7, Cor. 3.1, p. 335]). Hence $d^{1} \leq d_{s}$. Similar application of the Strum Separation Theorem to (14) and (16) now yields

$$
\lambda_{1}\left(B_{d_{s}}\right) \leq s+\lambda^{1} .
$$

Since we also have $\lambda_{1}\left(B_{d_{s}}\right)>\lambda_{1}\left(V_{d_{s}}\right)$, it follows from (7) and (19) that

$$
s+\lambda_{1}\left(V_{1-d_{s}}\right)=\lambda_{1}\left(V_{d_{s}}\right)<\lambda_{1}\left(B_{d_{s}}\right) \leq s+\lambda^{1} \leq s+\lambda_{1}\left(V_{1-d_{s}}\right),
$$

a contradiction which proves that $\lambda^{1}>\lambda_{1}\left(V_{1-d_{s}}\right)$.

Similarly as above, there exists $d^{2} \in(0,1)$ such that $v^{2}$ is a solution of

$$
\left\{\begin{array}{l}
v^{\prime \prime}+\frac{N-1}{r} v^{\prime}+\lambda^{2} v=0 \text { and } \quad v<0 \quad \text { in }\left(0, d^{2}\right) \\
v^{\prime}(0)=v\left(d^{2}\right)=0
\end{array}\right.
$$

and

$$
\left\{\begin{array}{l}
v^{\prime \prime}+\frac{N-1}{r} v^{\prime}+\left(s+\lambda^{2}\right) v=0 \quad \text { and } \quad v>0 \quad \text { in }\left(d^{2}, 1\right) \\
v\left(d^{2}\right)=v(1)=0
\end{array}\right.
$$

After the substitution $\hat{v}^{2}(r)=r^{\frac{1}{2}(N-1)} v^{2}(r), \hat{v}^{2}$ is a solution of

$$
\left\{\begin{array}{l}
\hat{v}^{\prime \prime}+\left(\lambda^{2}+\frac{(N-1)(3-N)}{4 r^{2}}\right) \hat{v}=0 \quad \text { and } \hat{v}<0 \quad \text { in }\left(0, d^{2}\right) \\
\hat{v}(0)=\hat{v}\left(d^{2}\right)=0
\end{array}\right.
$$

and

$$
\left\{\begin{array}{l}
\hat{v}^{\prime \prime}+\left(s+\lambda^{2}+\frac{(N-1)(3-N)}{4 r^{2}}\right) \hat{v}=0 \quad \text { and } \hat{v}>0 \quad \text { in }\left(d^{2}, 1\right) \\
\hat{v}\left(d^{2}\right)=\hat{v}(1)=0
\end{array}\right.
$$

Assume that $\lambda^{2} \leq \lambda_{1}\left(V_{1-d_{s}}\right)\left(<\lambda_{1}\left(B_{1-d_{s}}\right)\right)$ and that $1-d_{s}>d^{2}$. Similar arguments based on the Sturm Comparison Theorem yield first that $1-d_{s} \leq d^{2}$ (i.e., $1-d^{2} \leq d_{s}$ ), and then (16), (21) that

$$
\lambda_{1}\left(B_{d_{s}}\right) \leq s+\lambda^{2}
$$

As above we obtain

$$
s+\lambda_{1}\left(V_{1-d_{s}}\right)=\lambda_{1}\left(V_{d_{s}}\right)<\lambda_{1}\left(B_{d_{s}}\right) \leq s+\lambda^{2} \leq s+\lambda_{1}\left(V_{1-d_{s}}\right),
$$

a contradiction which proves that $\lambda^{2}>\lambda_{1}\left(V_{1-d_{s}}\right)$.

The assertion now follows from Proposition 5 .

Remark 7. Careful investigation of the above proof indicates that $(N-1)(3-N) \leq 0$ is needed to make the comparison arguments work. The proof is simpler for $N=3$ 
when the transformed equations for $\hat{v}$ and $\hat{w}$ are autonomous. The application of the Sturm Comparison Theorem is then more straightforward.

Acknowledgments

Jiři Benedikt and Petr Girg were supported by the Project KONTAKT, ME 10093, Pavel Drábek was supported by the Project KONTAKT, ME 09109.

\section{Author details}

${ }^{1}$ Department of Mathematics, Faculty of Applied Sciences, University of West Bohemia, Univerzitnl 22, 306 14 Plzen̆, Czech Republic ${ }^{2}$ Department of Mathematics and N.T.I.S., Faculty of Applied Sciences, University of West Bohemia, UniverzitnÍ 22, 30614 Plzeň, Czech Republic

\section{Authors' contribution}

All authors contributed to each part of this work equally.

\section{Competing interests}

The authors declare that they have no competing interests.

Received: 3 May 2011 Accepted: 4 October 2011 Published: 4 October 2011

\section{References}

1. Bartsch, T, Weth, T, Willem, M: Partial symmetry of least energy nodal solutions to some variational problems. J. D'Analyse Mathématique. 96, 1-18 (2005)

2. de Figueiredo, D, Gossez, J-P: On the first curve of the Fučík spectrum of an elliptic operator. Differ. Integral Equ. 7 1285-1302 (1994)

3. Bartsch, T, Degiovanni, M: Nodal solutions of nonlinear elliptic Dirichlet problems on radial domains. Rend. Licei Mat. Appl. 17, 69-85 (2006)

4. Cuesta, M, de Figueiredo, D, Gossez, J-P: The beginning of the Fučík spectrum for the p-Laplacian. J. Differ. Equ. 159, 212-238 (1999). doi:10.1006/jdeq.1999.3645

5. Takáč, P: Degenerate elliptic equations in ordered Banach spaces and applications. In: Drábek P, Krejčí P, Takáč P (eds.) Nonlinear Differential Equations. Chapman and Hall/CRC Res. Notes Math, vol. 404, pp. 111-196. CRC Press LLC, Boca Raton (1999)

6. Arias, M, Campos, J: Radial Fučik spectrum of the Laplace operator. J. Math. Anal. Appl. 190, 654-666 (1995). doi:10.1006/jmaa.1995.1101

7. Hartman, P: Ordinary Differential Equations. Wiley, New York (1964)

doi:10.1186/1687-2770-2011-27

Cite this article as: Benedikt et al:: The first nontrivial curve in the fučlk spectrum of the dirichlet laplacian on the ball consists of nonradial eigenvalues. Boundary Value Problems 2011 2011:27.

\section{Submit your manuscript to a SpringerOpen ${ }^{\circ}$ journal and benefit from:}

- Convenient online submission

Rigorous peer review

- Immediate publication on acceptance

- Open access: articles freely available online

- High visibility within the field

- Retaining the copyright to your article

Submit your next manuscript at $>$ springeropen.com 